\section{Total remission of severe immune thrombocytopenia after short term treatment with romiplostim}

\author{
Efthymia Vlachaki, Vaia Papageorgiou, \\ Filippos Klonizakis, Maria Spandonidou, \\ Sofia Chisan, Evaggelia Vetsiou, \\ Elissavet loannidou \\ Second Department of Internal Medicine, \\ Hematological Laboratory, Aristotle \\ University, Hippokrateon General \\ Hospital, Thessaloniki, Greece
}

\section{Abstract}

Immune thrombocytopenia (ITP) in adults is an acquired chronic immune-mediated disorder defined by isolated thrombocytopenia. In recent years, an improved understanding of the pathophysiology of ITP has been achieved and it is now accepted that the disorder is due to increased platelet destruction and decreased platelet production from megakaryocytes. Thrombopoietin (TP0)-receptor agonists (romiplostim and eltrombopag) are new therapeutic modalities in the treatment of ITP. Here we describe a case of an elderly patient with severe ITP who presented complete remission after short-term use of romiplostim (only 3 weekly doses). This finding is quite interesting as the TP0-receptor agonists are, so far, believed to rarely lead to off-treatment sustained remission. The common notion of long-term use of romiplostim could be reexamined in future studies. Furthermore, the short term treatment with romiplostim may reduce the cost and the risk of side effects.

\section{Introduction}

Immune thrombocytopenia (ITP) is an autoimmune syndrome involving antibodyand cell-mediated destruction of platelets and suppression of platelet production that may predispose to bleeding. Estimates of the incidence of adult-onset ITP range from approximately 1.6 to 3.9 per 100,000 persons per year, with a prevalence ranging from 9.5 to 23.6 per 100,000 persons, based on diagnostic codes in the UK health registry. ITP is generally a diagnosis of exclusion and in adults typically shows an insidious onset and follows a chronic course. ${ }^{1,2}$ In this case report, the first in the literature to our knowledge, we describe an 83year-old man who responded after short-term therapy with the TP0-receptor agonist romiplostim with complete remission, whereas pre- vious first-line interventions had not achieved response.

\section{Case Report}

An 83-year-old Caucasian male was admitted to our clinic because of sudden onset of gastrointestinal bleeding. His medical history included diagnosis of Rendu-Osler-Weber disease and a hospitalization for ITP 2 years before previous admission, successfully treated with corticosteroids. On physical examination the patient had no bruises or petechiae, was afebrile, his pulse was regular and his blood pressure and respiratory rate were normal. Clear lung sounds were found bilaterally. No focal neurologic deficits were found. In general, the patient was appropriately alert and oriented.

Laboratory studies were ordered and revealed thrombocytopenia and anemia (PLT: 8 $\times 10^{9} / \mathrm{L}, \mathrm{Hb} 10.5 \mathrm{~g} / \mathrm{dL}$ ). The peripheral blood smear showed thrombocytopenia without any morphological abnormalities. Additionally, immunologic and virology tests were carried out revealing no abnormality.

The patient was first treated with coricosteroids (prednisolone $1 \mathrm{mg} / \mathrm{kg}$ ) but without any response. A bone marrow aspiration and biopsy were performed with results compatible with ITP.

The patient's bleeding symptoms worsened and now also included hematuria and vesicular bleeding. Combined prednisolone and intravenous immunoglobulin (IVIg $400 \mathrm{mg} / \mathrm{kg}$ for 4 days) was then administered, with no response. Platelet transfusion was given, in an attempt to raise the platelet count quickly, as indicated in cases of emergency bleeding. The platelet count still did not increase at all. Rituximab, a monoclonal antibody against Bcell antigen CD-20, $\left(375 \mathrm{mg} / \mathrm{m}^{2}\right)$ was given in combination to corticosteroids, but still without success. Splenectomy was not an option due to the patient's refusal to undergo the specific procedure. Romiplostim (1 $\mu \mathrm{g} / \mathrm{kg}$ of a weekly subcutaneous injection) in combination with prednisolone ( $50 \mathrm{mg} /$ day) was decided. On the $3^{\text {rd }}$ weekly dose $(3 \mathrm{mg} / \mathrm{kg})$ the platelet count increased up to $100 \times 10^{9} / \mathrm{L}$. The $4^{\text {th }}$ dose was postponed since the platelet count was $700 \times 10^{9} / \mathrm{L}$ (Figure 1). Acetylsalicylic acid was added as a thromboprophylactic measure and the patient was then discharged. After 3 months of follow up (under corticosteroid therapy in the tapering phase, ie: $4 \mathrm{mg}$ of methylprednisolone day after day) the platelet count was still normal, at a level of approximately $400 \times 10^{9} / \mathrm{L}$. The patient remained in complete remission during the one year follow up.
Correspondence: Vlachaki Efthymia, Second Department of Internal Medicine, Hematological Laboratory, Aristotle University, Hippokrateon General Hospital, Amorgou 29 street, 55337, Thessaloniki, Greece.

Telefax: +30.2310 .857111 .

E-mail: efivlachaki@yahoo.gr

Key words: immune thrombocytopenia, thrombopoietin-receptor agonists, romiplostim.

Contributions: EV, VP data analysis and interpretation, literature reviewing, manuscript writing; FK, MS, SC, EV, data analysis and interpretation; $\mathrm{EI}$, literature reviewing and manuscript editing.

Conflict of interest: the authors report no conflicts of interest.

Received for publication: 26 July 2011.

Revision received: 12 October 2011.

Accepted for publication: 12 October 2011.

This work is licensed under a Creative Commons Attribution NonCommercial 3.0 License (CC BYNC 3.0).

(C) Copyright E. Vlachaki et al., 2011

Licensee PAGEPress, Italy

Hematology Reports 2011; 3:e20

doi:10.4081/hr.2011.e20

\section{Discussion}

Immune thrombocytopenia (ITP) is an acquired autoimmune disorder characterized by isolated thrombocytopenia, defined as a peripheral blood platelet count less than $100 \times 10^{9} / \mathrm{L}$, and the absence of any obvious initiating and/or underlying cause of thrombocytopenia. ITP is classified by duration into: newly diagnosed (diagnosis to 3 months), persistent (3-12 months' duration) and chronic (>12 months' duration). Severe ITP is defined as bleeding at presentation or during treatment requiring additional therapy. Refractory ITP is defined as the presence of severe ITP after splenectomy. Non-splenectomized patients are defined as responders or nonresponders to various treatments. ${ }^{3}$

Signs and symptoms can vary widely. In some cases ITP is asymptomatic, whereas in others development of visible signs such as bruises, petechiae, epistaxis or serious bleeding episodes occur (gastrointestinal hemorrhage, skin or mucosal hemorrhage, intracranial hemorrhage). The severity of thrombocytopenia seems to correlate to some extent with the bleeding risk. ${ }^{4}$

The pathogenesis of ITP is based on a combination of enhanced platelet clearance and a variably impaired platelet production.5,6 The disorder is due to a diversity of immune effects, so no single therapeutic approach is 


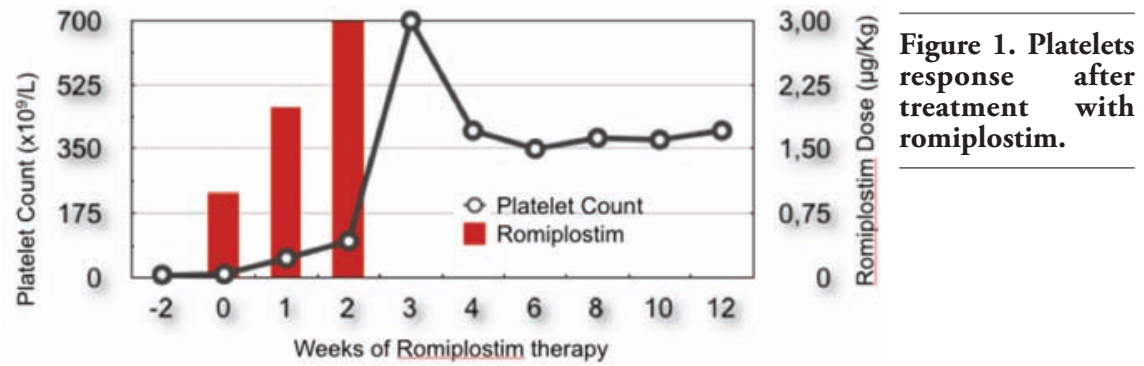

effective for all patients with ITP. Variations in the etiology of the disorder explain why some patients respond to therapy that suppresses Bcells, others to drugs that suppress T-cells and others to agents that activate thrombopoiesis. ${ }^{7-9}$ The TP0-receptor agonists (romiplostim and eltrombopag) are new therapeutic modalities that have recently been licenced for the treatment of ITP. ${ }^{10-13}$ Reported adverse effects of these agents have been relatively mild, although rare serious events such as bone marrow reticulin formation, thromboembolic episodes and liver function test abnormalities have occurred. Romiplostim and eltrombopag bear no structural homology to TPO but can both bind and activate the TPO receptor. Up to now, TPO-receptor agonists are used for patients at risk of bleeding, who relapse after splenectomy or who have a contraindication to splenectomy and who have already failed at least one other therapy. ${ }^{14}$ They are a costly option, but in view of the good tolerability and low toxicity they are often chosen for treatment.

In our case the patient was treated with romiplostim, achieved complete response after short term administration of the drug (only 3 weekly doses) and overall tolerated treatment very well. Since then he remains free of symptoms, with a stable and normal platelet count. This finding is quite interesting as the TPOreceptor agonists are, so far, believed to lead to off-treatment sustained remission very rarely. The common notion of long-term use of romiplostim could be re-examined in future studies It is worth mentioning that the successful response to romiplostim in our case may be explained by pre-stimulated thrombopoiesis as a result of the previous corticosteroid, IVIg and rituximab treatment. The pre-treatment with rituximab may be actually responsible for the excellent response to romiplostim. In recent bibliographic data, two cases have been described with effective treatment of severe ITP by combined rituximab and romiplostim. ${ }^{15}$ Our case accords with these findings with the difference that the patient received only one dose of rituximab and 3 doses of romiplostim afterwards.

Despite the plethora of therapeutic strategies of ITP there is no evidence to guide an algorithm of treatment for recurrent or persistent cases of ITP. According to the 2011 evidence based practice guidelines of the American Society of Hematology for ITP, additional studies are needed concerning the firstline therapy for the management of bleeding and the second-line therapies. ${ }^{14}$ If treatment is required it should be individualized for each patient and the final judgment should be made after thorough investigation of individual circumstances. The main goal is to achieve an increase of the platelet count that is hemostatic rather than normal.

The role of TPO agonists in the management of ITP will evolve in the future when results from clinical trials and experience will finally clarify their efficacy and potential side effects. For this purpose collaboration between health care centers is imperative. In this way the treatment of ITP will transform from opinion based to evidence-based. Nowadays, romiplostim seems a very promising therapy for the treatment of refractory forms of ITP so more clinical trials are needed in order to clarify the duration, dosage, effectiveness and safety of the drug either as monotherapy or combination therapy with other agents.

\section{References}

1. Provan D, Stasi R, Newland A, et al. International consensus report on the investigation and management of primary immune thrombocytopenia. Blood 2010; 115:168-86.

2. Cuker A, Cines DB. Immune thrombocytopenia. Hematology Am Soc Hematol Educ Program 2010;2010:377-84.

3. Rodeghiero F, Stasi R, Gernsheimer T, et al. Standardization of terminology, definitions and outcome criteria in immune thrombocytopenic purpura of adults and children: report from an international working group. Blood 2009;113:2386-93.

4. Neylon A, Saunders P, Howard M, et al. Clinically significant newly presenting autoimmune thrombocytopenic purpura in adults: a prospective study of a populationbased cohort of 245 patients. B J Haematol 2003;122:966-74.

5. Stasi R, Evangelista M, Stipa E, et al. Idiopathic thrombocytopenic purpura: current concepts in pathophysiology and management. Thromb Haemost 2008;99:4-13.

6. McMillan R. The pathogenesis of chronic immune thrombocytopenic purpura. Semin Hematol 2007 ;44Suppl5:S3-S11.

7. De Sauvage FJ, Hass PE, Spencer SD, et al. Stimulation of megakaryocytopoiesis and thrombopoiesis by the c-Mpl ligand. Nature 1994;369:533-8.

8. Kaushansky K, Lok S, HollY R, et al. Promotion of megakaryocyte progenitor expansion and differentiation by the $\mathrm{c}-\mathrm{Mpl}$ ligand thrombopoietin. Nature 1994; 369:568-71.

9. Mukai H, Kojima H, Todokoro K, et al. Serum thrombopoietin (TP0) levels in patients with amegakaryocytic thrombocytopenia are much higher than those with immune thrombocytopenic purpura. Thromb Haemost.1996;76:675-8.

10. Andemariam B, Psaila B, Bussel J. Novel thrombopoietic agents. Hematol Am Soc Hematol Educ Program 2007;106-13.

11. Cersosimo R. Romiplostim in chronic immune thrombocytopenic purpura. Clin Ther 2009;31:1887-907.

12. Kuter DJ, Bussel JB, Lyons RM, et al. Efficacy of romiplostim in patients with chronic immune thrombocytopenic purpura: a double-blind randomised controlled trial. Lancet 2008;371:395-403.

13. Bussel JB, Kuter DJ, George JN, et al. AMG 531, a thrombopoiesis-stimulating protein, for chronic ITP. N Engl J Med 2006;355: 1672-81.

14. Neunert C, Lim W, Crowther M, et al. The American Society of Hematology 2011 evidence-based practice guideline for immune thrombocytopenia. Blood 2011; 117:4190-207.

15. Pohlen M, Sargin B, Zicholl S, et al. Combination of romiplostim and rituximab: effective therapy of severe immune thrombocytopenia. Eur J Haematol 2010; 84:362-4. 